\title{
The Effective Diffusivity in Two-Phase Material
}

\author{
I V Belova and G E Murch
}

\author{
Diffusion in Solids Group \\ School of Engineering \\ The University of Newcastle, Callaghan \\ New South Wales 2308 \\ Australia
}

Keywords: Diffusivity, Diffusion Coefficient, Two-phase, Monte Carlo

\begin{abstract}
In this paper we employ Monte Carlo computer simulation to test the extended Maxwell-Garnett expression for the effective diffusivity in a simple model of a two phase material. We determine the effective diffusivity in f.c.c., b.c.c. and s.c. type arrangements of dispersed spheres at variable densities and for cases where the diffusivity in the dispersed phase was less than, and greater than, the diffusivity in the matrix phase. It is shown that the above equation agrees very well with the simulation data for all densities up to where the spheres of the dispersed phase touch and over six orders of magnitude in the ratio of the diffusivity of the dispersed phase to the diffusivity of the matrix phase.
\end{abstract}

\section{Introduction}

A long-standing problem in the area of diffusion in solids is the determination of accurate expressions for the effective diffusivity $\mathrm{D}_{\text {eff }}$ in two-phase material given the individual diffusivities in the component phases. We are referring here to diffusion that does not alter the morphology or growth of the two phases during the diffusion time. Microscopic examples might include the tracer diffusion of a host component or an impurity in a stable two-phase alloy, or the (interstitial) permeation of hydrogen through a stable two-phase alloy. At low temperatures we are, of course, likely to encounter principally short-circuit diffusion along the interphase boundaries. At high temperatures, however, we can expect that lattice diffusion will prevail but proceed through each phase at different rates. There will be an overall 'effective' bulk diffusivity that is dependent on the relative amounts and the morphology of the two phases.

In this paper we will refer to the host or matrix phase as phase 1 and the dispersed phase as phase 2. Much of the older literature on the subject deals with a diffusant in the pore space of an impermeable second phase, usually represented as spheres, see, for example, the review by German [1]. This is a very well-studied special case of diffusion in two-phase material. Maxwell [2] derived the following classic expression relating the effective diffusivity $\mathrm{D}_{\mathrm{eff}}$ of the diffusant when exploring the pore space:

$$
\mathrm{D}_{\text {eff }}=\frac{2 \mathrm{D}_{1} \varepsilon}{3-\varepsilon}
$$

where the pore fraction is given by $\varepsilon$ and $D_{1}$ is the diffusivity in the absence of the impermeable phase. Eq. 1 was originally derived for the limiting case $\varepsilon \rightarrow 1$. Neale and Bader [3] derived it once again for an idealized geometric model for use over the entire porosity range. It has subsequently been shown by Hashkin and Shtrikman [4] that Eq. 1 represents the upper bound for $\mathrm{D}_{\text {eff }} / \mathrm{D}_{1}$ for any isotropic medium for all $\varepsilon$, even when spheres do not represent the impermeable phase. Using a minimum entropy argument Prager [5] derived the following condition for $\mathrm{D}_{\text {eff }} / \mathrm{D}_{1}$ for particles of arbitrary shape: 


$$
\mathrm{D}_{\text {eff }}<\varepsilon \mathrm{D}_{1}\left(1-\frac{1-\varepsilon}{3}\right)
$$

and for spheres in particular:

$$
\mathrm{D}_{\text {eff }}=\varepsilon \mathrm{D}_{1}\left(1-\frac{1-\varepsilon}{2}\right)
$$

Bruggemann [6] studied a system where one large sphere is surrounded by a homogeneous distribution of much smaller spheres. Assuming that the system is very dilute in large spheres Bruggemann adjusted the Maxwell result for the limit $\varepsilon \rightarrow 1$ to give:

$$
\mathrm{D}_{\text {eff }}=\mathrm{D}_{1} \varepsilon^{3 / 2}
$$

Maxwell-Garnett [7] extended Eq. 1 to include a non-zero diffusivity $\mathrm{D}_{2}$ in the dispersed phase (phase 2):

$$
D_{\text {eff }}=D_{1}\left[1+\frac{d\left(D_{2}-D_{1}\right)(1-\varepsilon)}{D_{2}+(d-1) D_{1}-\left(D_{2}-D_{1}\right)(1-\varepsilon)}\right]
$$

where $d$ is the dimension $(d=1,2$ or 3). (Maxwell-Garnett's original equation put $d=3$.)

More recently, using a concentric sphere model and phenomenological diffusion arguments Kalnin et al. [7-9] developed the Maxwell-Garnett equation (Eq. 5) further to take into account the possible unequal partitioning of the diffusant between the two phases. Eq.5 then becomes:

$$
\mathrm{D}_{\text {eff }}=\frac{\mathrm{sD}_{1}\left[(\varepsilon+\mathrm{d}-\varepsilon \mathrm{d}) \mathrm{D}_{2}+\mathrm{s} \varepsilon \mathrm{D}_{1}(\mathrm{~d}-1)\right]}{(1-\varepsilon+\mathrm{s} \varepsilon)\left[\mathrm{sD}_{1}(\mathrm{~d}-\varepsilon)+\varepsilon \mathrm{D}_{2}\right]}
$$

where $\mathrm{s}$ is the segregation factor or segregation coefficient and is defined as the equilibrium concentration of diffusant in phase 1 to the equilibrium concentration of diffusant in phase 2 . Note that except for the case when $\mathrm{d}=1$ (then the two phases simply alternate in one direction), Eqs 5 and 6 are not symmetric with respect to the interchange of phases 1 and 2 . Put another way, as written, Eqs 5 and 6 refer to a situation only where phase 2 is the dispersed phase.

Eq. 6 can be made to reduce to Eq. 1 by putting $d=3$ and setting $\mathrm{D}_{2}=0$ and $\mathrm{s}=1$. It may appear inconsistent with the notion of an impermeable second phase to put the diffusivity equal to zero in this phase yet assume that the concentration of diffusant is equal in both phases $(s=1)$ [8-10]. It would seem much more appropriate to put $\mathrm{s}^{-1}=0$ (and $\mathrm{D}_{2}=0$ ). If one does this, it is found that Eq. 1 is in fact recovered but without the $\varepsilon$ in the numerator. This is in fact correct. The apparent discrepancy comes about because in the above studies of diffusion in the presence of an impermeable phase, $\mathrm{D}_{\text {eff }}$ was defined by way of Fick's First Law in such a way that the impermeable phase was not considered part of the accessible structure. For comparison purposes with the other equations given in this paper it is necessary then to divide the RHS of Eqs $1-4$ by a factor $\varepsilon$.

The major assumption in Eqs 5 and 6 is that it refers to a hypothetical diffusion situation where the two phases are arranged alternately in all directions. Eqs 5 and 6 do not contain information about the actual arrangement or morphology of the second phase. Eq. 6 has been tested by Monte Carlo simulation for the case of impermeable circles in 2D that were mapped onto hexagonal and square lattices [8-10]. The agreement was very good. Similarly, 
a preliminary study of diffusion in random packing arrangements of impermeable spheres of different sizes also showed satisfactory agreement (actually with Eq. 1) [11].

For completeness in our discussion we make mention of the extended Hart-Mortlock Equation for describing $D_{\text {eff. }}$ This equation was originally derived for the case of tracer diffusion in the presence of parallel short circuit paths by Hart [12], extended by Mortlock [13] to include segregation, and finally made rigorous for the case of diffusion in one dimension by Kaur et al. [14]. The major assumption here is that diffusion proceeds along entirely parallel paths. The extended Hart-Mortlock Equation reads:

$$
\mathrm{D}_{\text {eff }}=\frac{\mathrm{s} \varepsilon \mathrm{D}_{1}+(1-\varepsilon) \mathrm{D}_{2}}{1-\varepsilon+\mathrm{s} \varepsilon}
$$

Monte Carlo studies have shown that Eq. 7 by itself provides a rather poor description of the effective diffusivity at more than very low densities of the dispersed phase [15-17].

The extended Maxwell-Garnett Equation i.e. Eq. 6 appears to be a potentially very useful general expression for describing the effective diffusivity in two-phase material. Apart from the Monte Carlo studies mentioned above for testing it, there have been several other Monte Carlo studies of the effective diffusivity in the presence of squares [15,16] and cubes [17] in various ordered arrangements and with differing diffusivities. These studies were concerned with diffusion in single phase nanocrystalline material where the grain boundaries (at high densities) can be regarded as a separate high diffusivity 'phase'. The possibility of segregation was also permitted. These studies have shown that, depending on density, combinations of Eqs 6 and 7 that take into account the actual arrangements perform better than the basic Eq. 6 itself. A Monte Carlo study of the case of ordered mono-spheres as the dispersed phase and at varying densities with varying diffusivities would be useful for the further testing of Eq. 6. This model is a useful one for describing diffusion in two-phase structures where the second phase is dispersed in the matrix of the first. In the present paper we undertake a systematic Monte Carlo study to calculate the effective diffusivity in ordered (f.c.c., b.c.c. and s.c.) arrays of mono-spheres.

\section{Monte Carlo Simulation}

In order to attack the problem we represented the diffusivities in the two individual phases by making use of the equivalence of the Fick and Einstein definitions of the diffusivity. In particular, we mapped a periodic unit cell of the ordered arrangement (f.c.c., b.c.c. or s.c.) of spheres onto a very fine mesh array $(600 \times 600 \times 600)$ that was then explored by randomly walking particles released one at a time and directed by standard Monte Carlo computer simulation methods, see, for example, refs 18,19 . The array was, in effect, a fine-grained replica of the original spatial arrangement of the spheres. This array has no atomistic meaning: it is purely an artifice to enable the macroscopic diffusivity to be represented by means of a diffusivity based on a random walk of a particle on a lattice. This procedure has been used many times in related problems, especially in connection with grain boundary diffusion, see, for example, ref. 19. The diffusivities were scaled directly to the jump

frequencies from site to site in each phase, time was scaled to the number of jump attempts of a particle. The segregation factor s was put equal to unity. The particles were released from randomly chosen sites in the array when $s=1$. (If one wishes to study, say, $s \neq 1$ then the type of starting site can be weighted accordingly.) It was found that several thousand attempts were sufficient to converge to a diffusivity as determined by the Einstein equation:

$$
\mathrm{D}=\frac{<\mathrm{R}^{2}>}{6 \mathrm{t}}
$$


where $\mathrm{R}$ is the displacement of a particle after time $\mathrm{t}$ and the Dirac brackets indicate an average over a large number of particles, in this case, $10^{6}$.

\section{Results and Discussion}

In Fig. 1 we present results for the normalized effective diffusivity as a function of the fraction (1-E) of phase 2 for the case of a f.c.c. type of arrangement of

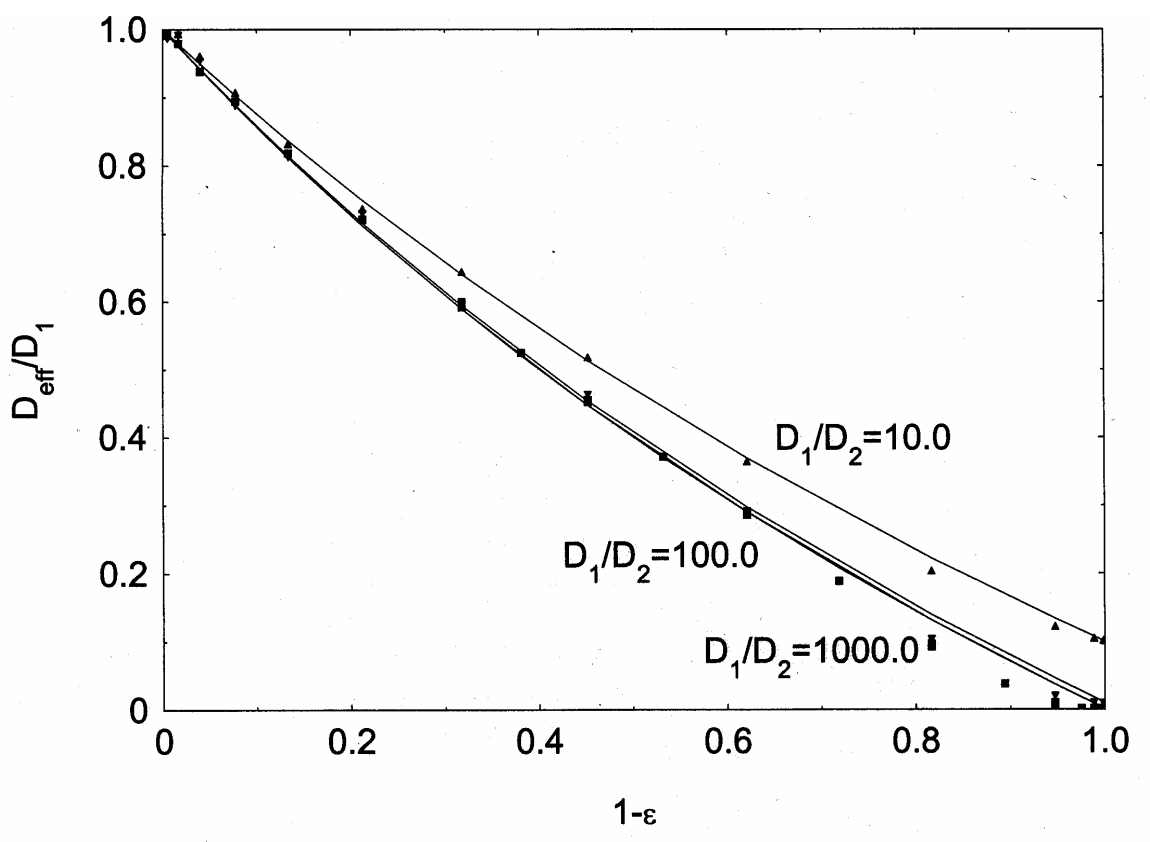

Figure 1. $D_{\text {eff }} / D_{1}$ as a function of the fraction $(1-\varepsilon)$ of phase 2 for the case of a f.c.c. type of arrangement of spheres for three ratios of $\mathrm{D}_{1} / \mathrm{D}_{2}$ : Monte Carlo simulation results, $\boldsymbol{\Delta}: \mathrm{D}_{1} / \mathrm{D}_{2}=10.0 ; \boldsymbol{\nabla}: \mathrm{D}_{1} / \mathrm{D}_{2}=100.0 ; \mathbf{\mathbf { m }}: \mathrm{D}_{1} / \mathrm{D}_{2}$ $=1000.0$. Solid lines correspond to Eq. 6 .

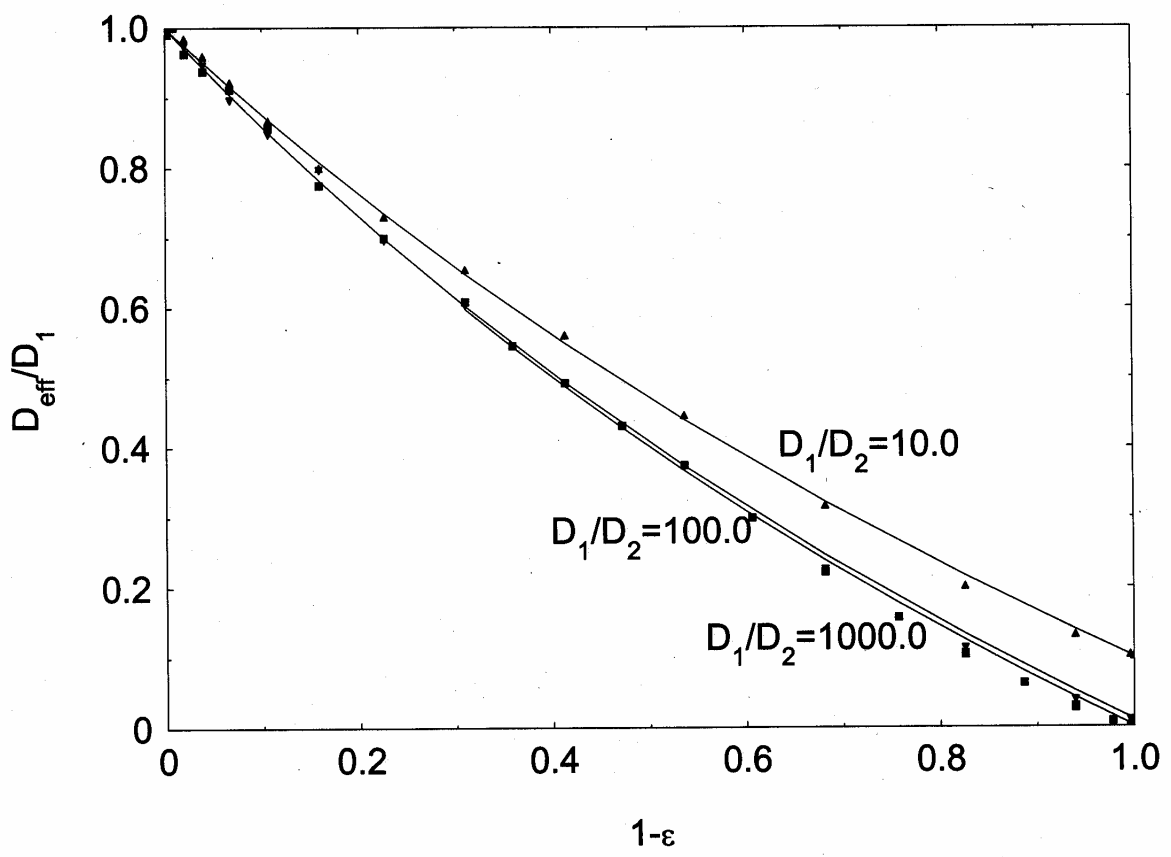

Figure 2. $\mathrm{D}_{\text {eff }} / \mathrm{D}_{1}$ as a function of the fraction $(1-\varepsilon)$ of phase 2 for the case of a b.c.c. type of arrangement of spheres for three ratios of $\mathrm{D}_{1} / \mathrm{D}_{2}$ : Monte Carlo simulation results, $\boldsymbol{\Delta}: \mathrm{D}_{1} / \mathrm{D}_{2}=10.0 ; \boldsymbol{\nabla}: \mathrm{D}_{1} / \mathrm{D}_{2}=100.0 ; \mathbf{\mathbf { m }}: \mathrm{D}_{1} / \mathrm{D}_{2}$ $=1000.0$. Solid lines correspond to Eq. 6 


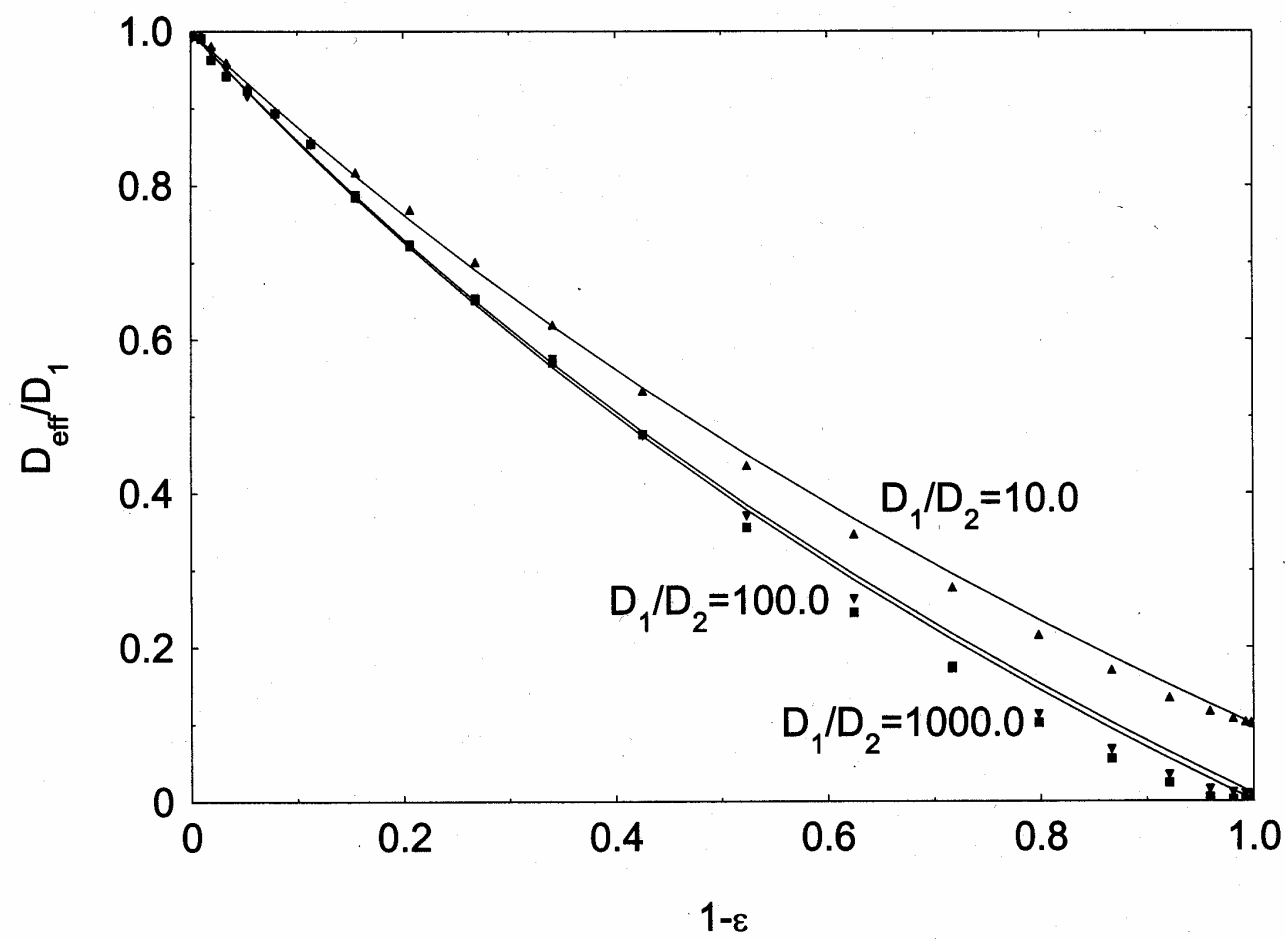

Figure 3. $D_{\text {eff }} / \mathrm{D}_{1}$ as a function of the fraction (1- $\varepsilon$ ) of phase 2 for the case of a s.c. type of arrangement of spheres for three ratios of $\mathrm{D}_{1} / \mathrm{D}_{2}$ : Monte Carlo simulation results, $\boldsymbol{\Delta}: \mathrm{D}_{1} / \mathrm{D}_{2}=10.0 ; \boldsymbol{\nabla}: \mathrm{D}_{1} / \mathrm{D}_{2}=100.0 ; \mathbf{\square}: \mathrm{D}_{1} / \mathrm{D}_{2}$ $=1000.0$. Solid lines correspond to Eq. 6 .

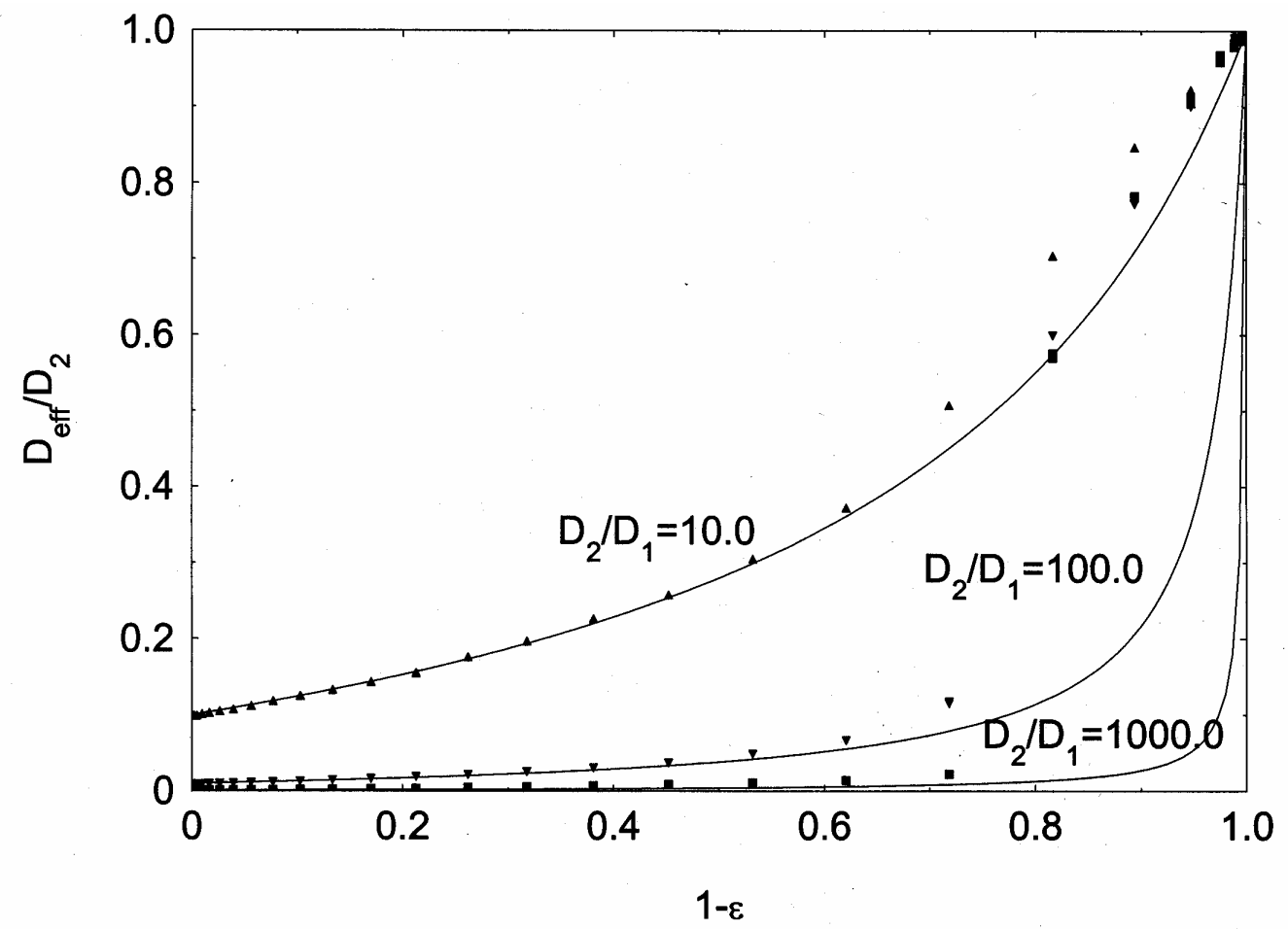

Figure 4. $\mathrm{D}_{\text {eff }} / \mathrm{D}_{2}$ as a function of the fraction $(1-\varepsilon)$ of phase 2 for the case of a f.c.c. type of arrangement of spheres for three ratios of $\mathrm{D}_{2} / \mathrm{D}_{1}$ : Monte Carlo simulation results, $\boldsymbol{\Delta}: \mathrm{D}_{2} / \mathrm{D}_{1}=10.0 ; \boldsymbol{\nabla}: \mathrm{D}_{2} / \mathrm{D}_{1}=100.0 ; \mathbf{m}: \mathrm{D}_{2} / \mathrm{D}_{1}$ $=1000.0$. Solid lines correspond to Eq. 6 . 


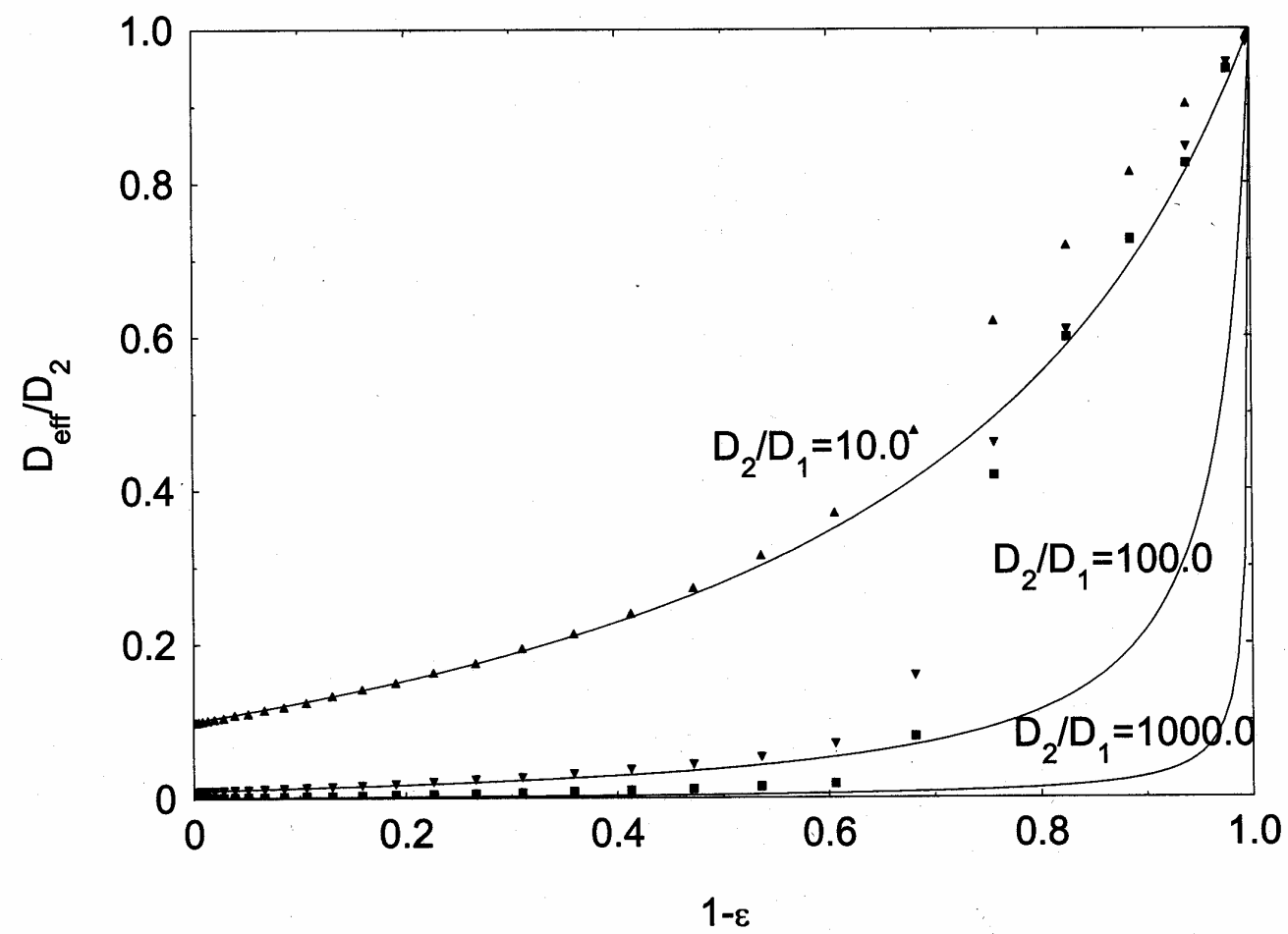

Figure 5. $D_{\text {eff }} / \mathrm{D}_{2}$ as a function of the fraction $(1-\varepsilon)$ of phase 2 for the case of a b.c.c. type of arrangement of spheres for three ratios of $\mathrm{D}_{2} / \mathrm{D}_{1}$ : Monte Carlo simulation results, $\boldsymbol{\Delta}: \mathrm{D}_{2} / \mathrm{D}_{1}=10.0 ; \boldsymbol{\nabla}: \mathrm{D}_{2} / \mathrm{D}_{1}=100.0 ; \mathbf{\mathbf { a }}: \mathrm{D}_{2} / \mathrm{D}_{1}$ $=1000.0$. Solid lines correspond to Eq. 6 .

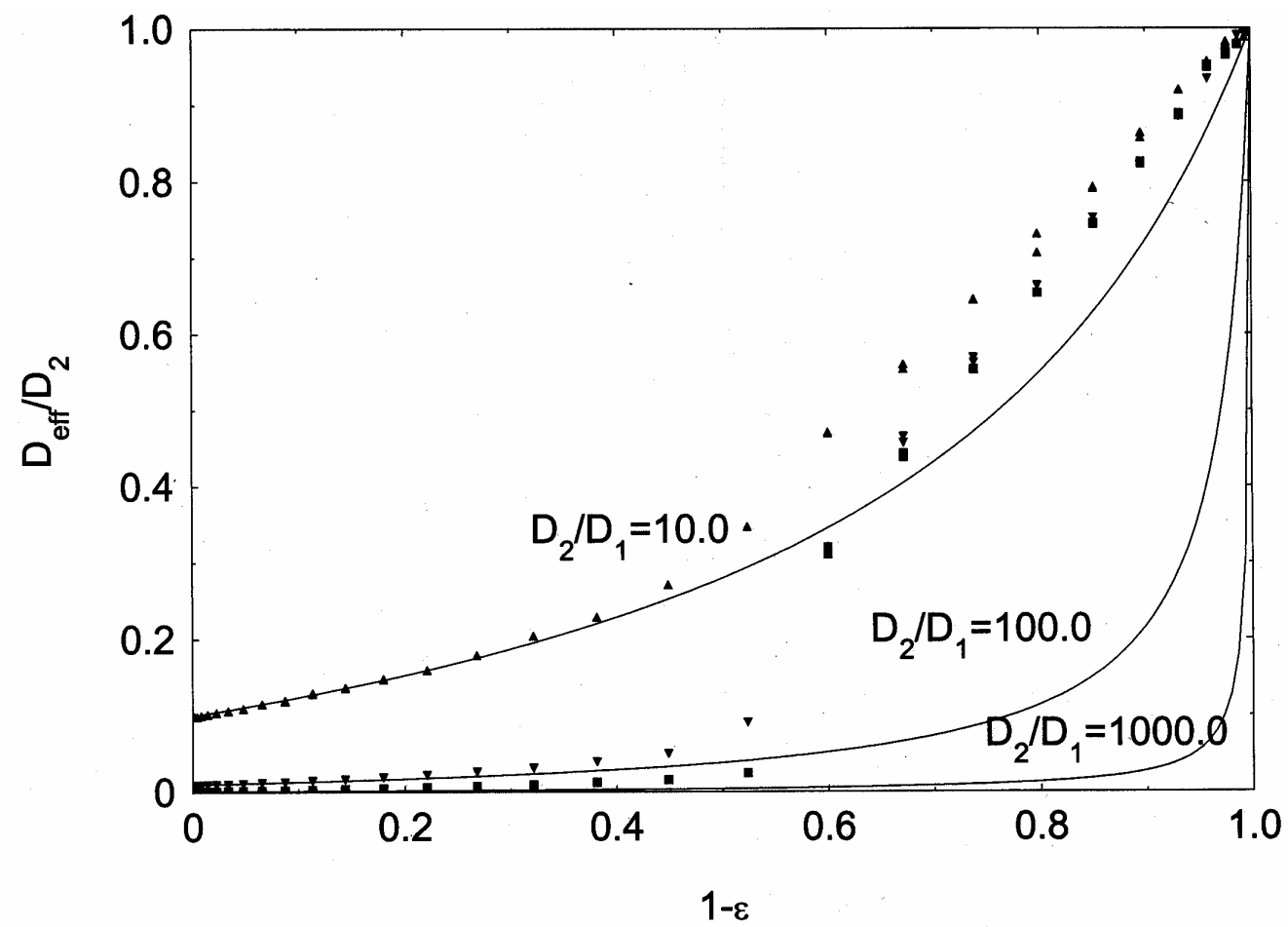

Figure 6. $\mathrm{D}_{\text {eff }} / \mathrm{D}_{2}$ as a function of the fraction (1- $\varepsilon$ ) of phase 2 for the case of a s.c. type of arrangement of spheres for three ratios of $\mathrm{D}_{2} / \mathrm{D}_{1}$ : Monte Carlo simulation results, $\boldsymbol{\Delta}: \mathrm{D}_{2} / \mathrm{D}_{1}=10.0 ; \boldsymbol{\nabla}: \mathrm{D}_{2} / \mathrm{D}_{1}=100.0 ; \mathbf{m}: \mathrm{D}_{2} / \mathrm{D}_{1}$ $=1000.0$. Solid lines correspond to Eq. 6 . 
spheres of phase 2 . Note that the entire density range is covered simply by allowing the spheres to overlap. Results for three ratios $(1000,100$, and 10) of the diffusivity in the matrix phase (phase 1) to that of the diffusivity in the dispersed phase (phase 2) are shown. The highest ratio gives a result that is very similar to the case of impermeable spheres [11]. It is clear that the extended Maxwell-Garnett equation (Eq. 6) does very well in its description of the effective diffusivity up to roughly the density fraction where the spheres touch, this is given by $(1-\varepsilon)=0.74$. Above this threshold the Monte Carlo results increasingly deviate from that predicted by Eq. 6. This deviation is of course not unexpected since Eq. 6 assumes that phase 2 is not continuous. In Figs 2 and 3 we show corresponding results for b.c.c. and s.c. type arrangements of the spheres respectively. These arrangements exhibit completely analogous behaviour to the f.c.c. case: the thresholds in the b.c.c. and s.c. cases are now at 0.68 and 0.524 respectively.

In Fig. 4 we present results for the converse situation where the dispersed phase (phase 2) in a f.c.c. type arrangement of spheres has a higher diffusivity than the matrix phase (phase 1). Results for three ratios (1000, 100 and 10) of the diffusivity in the dispersed phase to that of the diffusivity in the matrix are shown. It is clear that the extended MaxwellGarnett equation (Eq. 6) again does very well in its description of the effective diffusivity but there are deviations as the fraction where the spheres touch is approached. This fraction acts essentially like a percolation threshold in the sense that the effective diffusivity rapidly increases at this point where phase 2 becomes continuous. Eq. 6 does not contain the physics to explain this percolation behaviour. In Figs 5 and 6 we show corresponding results for b.c.c. and s.c. type arrangements of the spheres respectively. These arrangements exhibit completely analogous behaviour to the f.c.c. case but with different thresholds as given above.

It is clear from the present Monte Carlo study and the earlier ones [8-10] that the Maxwell-Garnett Equation does very well indeed for expressing the effective diffusivity in an assembly of spheres (and circles) for all densities up to where the spheres (and circles) touch. The actual type of arrangement has little apparent influence on this. On the other hand, as mentioned above, other Monte Carlo studies [15-17] have shown that the MaxwellGarnett Equation does not do so well when the dispersed phase is represented as squares or cubes as might be used as a primitive model for grains surrounded by wide grain boundary regions in nanocrystalline materials.

\section{Summary}

In this paper we have employed Monte Carlo computer simulation to test the extended Maxwell-Garnett expression (Eq. 6) for the effective diffusivity in a simple model of a twophase material. We determined the effective diffusivity in f.c.c., b.c.c. and s.c. type arrangements of spheres over a wide range of density and for cases where the diffusivity in the dispersed phase was less than, and greater than, the diffusivity in the matrix. It was shown that Eq. 6 agrees very well with the simulation data for all densities up to where the spheres of the dispersed phase touch, and over six orders of magnitude in the ratio of the diffusivity of the dispersed phase to the diffusivity of the matrix phase.

\section{Acknowledgements}

We would like to thank the Australian Research Council for its support of this work under the Large Grants and Discovery Project Grants Schemes. One of us (I.V.B.) wishes to thank the Australian Research Council for the award of a Queen Elizabeth II Fellowship. 


\section{References}

[1] R. M. German: Particle Packing Characteristics (Metal Powder Industries Federation, 1989).

[2] J.C.Maxwell, Treatise on Electricity and Magnetism (Clarendon Press, Oxford, 1904)

[3] G. H. Neale and W. K. Bader: AIChE Journal, Vol. 19 (1973) p. 112.

[4] Z. Hashkin and S. Shtrikman: J. Appl. Phys., Vol. 33 (1962) p. 3125.

[5] S. Prager: Physica, Vol. 29 (1963) p. 129.

[6] D. A. G. Bruggemann: Ann. Physik, Vol. 24 (1935) p. 636.

[7] J. C. Maxwell-Garnett: Philos. Trans. R. Soc. London., Vol. 203 (1904), p. 385.

[8] J. R. Kalnin and E. A. Kotomin: J. Phys. A: Gen. and Math., Vol. 31 (1998) p. 7227.

[9] J. R. Kalnin, E. A. Kotomin and J. Maier: J. Phys. Chem Solids, Vol. 63 (2002) p. 449.

[10] J. R. Kalnin, E. A. Kotomin and V. N. Kuzovkov: Def. Diffus. Forum, Vols 194199 (2001) p. 163.

[11] D. P. Riley, I. V. Belova and G. E. Murch: Mat. Res. Soc. Proc., Vol. 677 (2001) AA7.11.1 (electronically published).

[12] E. W. Hart: Acta Metall., Vol. 5 (1957) p. 597.

[13] A. J. Mortlock: Acta Metall., Vol. 8 (1962) p. 132.

[14] I. Kaur, Y. Mishin and W. Gust: Fundamentals of Grain and Interphase Boundary Diffusion, (Wiley, Chichester, 1995).

[15] I. V. Belova and G. E. Murch: J. Phys. Chem. Solids, in press.

[16] I. V. Belova and G. E. Murch: Mat. Res. Soc. Proc., Vol. 731 (2002) W5.5.1 (electronically published).

[17] I. V. Belova and G. E. Murch: Mass and Charge Transport in Inorganic Materials (CIMTEC2002) (Techna, Faenza, Italy, 2002) in press.

[18] G. E. Murch: Diffusion in Crystalline Solids, edited by G.E.Murch and A.S.Nowick (Academic, Orlando, 1984) p. 379.

[19] G. E. Murch and I. V. Belova: Interface Science, Vol. 11 (2003) p. 91.

\section{Erratum}

In Defect and Diffusion Forum Vols. 210-212 (2002), the title of the paper by I.V.Belova and G.E.Murch (p55) was inadvertently changed, during production, to "Tracer Diffusion in the Concentrated Lattice Gas with Self-Excitation". It should have been, "Tracer Diffusion in the Concentrated Lattice Gas with Self-Exclusion". My apologies for any confusion caused. 
Figure captions:

Figure 1. $D_{\text {eff }} / D_{1}$ as a function of the fraction (1- $\varepsilon$ ) of phase 2 for the case of a f.c.c. type of arrangement of spheres for three ratios of $\mathrm{D}_{1} / \mathrm{D}_{2}$ : Monte Carlo simulation results, $\boldsymbol{\Delta}: \mathrm{D}_{1} / \mathrm{D}_{2}=10.0 ; \boldsymbol{\nabla}: \mathrm{D}_{1} / \mathrm{D}_{2}=100.0 ; \mathbf{m}: \mathrm{D}_{1} / \mathrm{D}_{2}$ $=1000.0$. Solid lines correspond to Eq. 6 .

Figure 2. $\mathrm{D}_{\mathrm{eff}} / \mathrm{D}_{1}$ as a function of the fraction $(1-\varepsilon)$ of phase 2 for the case of a b.c.c. type of arrangement of spheres for three ratios of $\mathrm{D}_{1} / \mathrm{D}_{2}$ : Monte Carlo simulation results, $\boldsymbol{\Delta}: \mathrm{D}_{1} / \mathrm{D}_{2}=10.0 ; \boldsymbol{\nabla}: \mathrm{D}_{1} / \mathrm{D}_{2}=100.0 ; \mathbf{\mathbf { m }}: \mathrm{D}_{1} / \mathrm{D}_{2}$ $=1000.0$. Solid lines correspond to Eq. 6 .

Figure 3. $D_{\text {eff }} / D_{1}$ as a function of the fraction (1- $\varepsilon$ ) of phase 2 for the case of a s.c. type of arrangement of spheres for three ratios of $\mathrm{D}_{1} / \mathrm{D}_{2}$ : Monte Carlo simulation results, $\boldsymbol{\Lambda}: \mathrm{D}_{1} / \mathrm{D}_{2}=10.0 ; \boldsymbol{\nabla}: \mathrm{D}_{1} / \mathrm{D}_{2}=100.0 ; \mathbf{\square}: \mathrm{D}_{1} / \mathrm{D}_{2}$ $=1000.0$. Solid lines correspond to Eq. 6 .

Figure 4. $\mathrm{D}_{\text {eff }} / \mathrm{D}_{2}$ as a function of the fraction (1- $\varepsilon$ ) of phase 2 for the case of a f.c.c. type of arrangement of spheres for three ratios of $\mathrm{D}_{2} / \mathrm{D}_{1}$ : Monte Carlo simulation results, $\boldsymbol{\Delta}: \mathrm{D}_{2} / \mathrm{D}_{1}=10.0 ; \boldsymbol{\nabla}: \mathrm{D}_{2} / \mathrm{D}_{1}=100.0 ; \mathbf{m}: \mathrm{D}_{2} / \mathrm{D}_{1}$ $=1000.0$. Solid lines correspond to Eq. 6 .

Figure 5. $\mathrm{D}_{\text {eff }} / \mathrm{D}_{2}$ as a function of the fraction (1- $\varepsilon$ ) of phase 2 for the case of a b.c.c. type of arrangement of spheres for three ratios of $\mathrm{D}_{2} / \mathrm{D}_{1}$ : Monte Carlo simulation results, $\boldsymbol{\Lambda}: \mathrm{D}_{2} / \mathrm{D}_{1}=10.0 ; \boldsymbol{\nabla}: \mathrm{D}_{2} / \mathrm{D}_{1}=100.0 ; \mathbf{m}: \mathrm{D}_{2} / \mathrm{D}_{1}$ $=1000.0$. Solid lines correspond to Eq. 6 .

Figure 6. $D_{\text {eff }} / D_{2}$ as a function of the fraction (1- $\varepsilon$ ) of phase 2 for the case of a s.c. type of arrangement of spheres for three ratios of $\mathrm{D}_{2} / \mathrm{D}_{1}$ : Monte Carlo simulation results, $\boldsymbol{\Delta}: \mathrm{D}_{2} / \mathrm{D}_{1}=10.0 ; \boldsymbol{\nabla}: \mathrm{D}_{2} / \mathrm{D}_{1}=100.0 ; \mathbf{\mathbf { m }}: \mathrm{D}_{2} / \mathrm{D}_{1}$ $=1000.0$. Solid lines correspond to Eq. 6 . 
Defects and Diffusion in Ceramics

doi:10.4028/3-908450-81-0

The Effective Diffusivity in Two-Phase Material

doi:10.4028/3-908450-81-0.79 